\title{
Evaluación del programa de ejercicios físicos y educación diabeto- lógica en Danlí, Honduras
}

\author{
Raúl Orlando Figueroa Soriano ${ }^{1}$ \\ Jaime Neftalí Valerio Fortín ${ }^{2}$ \\ Miriam Ordoñez ${ }^{3}$
}

\section{RESUMEN}

Existen algunos estudios previos en donde el ejercicio físico se utilizó para controlar la diabetes mellitus II. En el año 2005, el Dr. Orestes demostró beneficios para controlar la glicemia en Cuba y en el mismo año, la Dra. Alicia Norma Alayón ejecutó rutinas aeróbicas para reducir la glicemia. En Honduras, el MSc. Tony Vega y Céleo Padilla realizaron un estudio descriptivo en el año 2012. El objetivo general del estudio fue evaluar los resultados de la aplicación del programa de ejercicios físicos y educación diabetológica para mejorar el control metabólico y calidad de vida de los pacientes diabéticos tipo II del Instituto Hondureño de Seguridad Social (IHSS) y del Hospital Regional Gabriela Alvarado (HRGA) de la ciudad de Danlí. El estudio se realizó con enfoque cuantitativo, casi experimental. Se estudiaron dos grupos: uno experimental y otro de control. Los criterios de inclusión utilizados fueron: residir en Danlí, disponibilidad de horario, carecer de morbilidad secundaria a diabetes mellitus, no padecer de artritis, ni artrosis osteoarticulares, ni fibromialgia, de edad comprendida entre los 40-75 años. La muestra era de tipo no probabilística por cuota; 30 personas del HRGA (experimental) y 30 personas del IHSS (control).

Los pacientes fueron evaluados por personal de cultura física, Medicina, Enfermería, Psicología, Nutrición y Estadística, durante un periodo de 18 semanas con tres seguimientos semanales. Se controlaban los niveles de glicemia, presión arterial, pulso, frecuencia cardiaca, saturación de oxígeno, peso, y estado psicológico. Las pruebas estadísticas realizadas comprendían las medidas de tendencia central, dispersión, y T de student. Los resultados indicaron: disminución de glucosa, hemoglobina glucosi-

1 Beneficiario de beca sustantiva de la DICYP, profesor del Departamento de Humanidades y Artes, Tec-Danlí, UNAH: pitolamaquina2@hotmail.com

2 Beneficiario de beca sustantiva de la DICYP, profesor del Departamento de Ciencias Económicas, Tec-Danlí, UNAH: jaimevale@hotmail.com

${ }^{3}$ Beneficiaria de beca sustantiva de la DICYP, profesor del Departamento de Ciencias Médicas,

Tec-Danlí, UNAH: Miriam.ordoñez79@unah.edu.hn 
lada, del LDL, triglicéridos, colesterol total, ácido úrico, masa corporal, índice de cintura cadera y aumento de la hemoglobina en el post test. El VO2 Max presentó un aumento significativo, disminuyendo el Índice de Rufier. La rapidez de pensamiento y la autoestima mejoró en los pacientes post intervención.

Palabras clave: Diabetes mellitus, ejercicio físico, calidad de vida.

\section{ABSTRACT}

Some previous studies exist where physical exercise was used for controlling the diabetes mellitus type II. In the year 2005, Dr. Orestes demonstrated benefits to control blood sugar in Cuba and in the same year, Dra. Alicia Norma Alayón executed aerobic routines to reduce the blood sugar. In Honduras, the MSc. Tony Vega and Celeo Padilla realized a descriptive study in the year 2012. The general aim of the study was to evaluate the results of the application of the program of physical exercises and diabetes education to improve the metabolic control and quality of life of the diabetic patients type II in the Honduran Institute of National Health Service (IHSS) and the Regional Hospital Gabriela Alvarado (HRGA) of Danli's city. The study was realized by quantitative approach, semi experimental.

Two groups were studied: the experimental and different one of control. The used criteria of incorporation were: to reside in Danlí, availability of schedule, to lack secondary morbidity to diabetes mellitus, to suffer neither from arthritis, nor degenerative osteoarthritis osteoarticulares, nor fibromyalgia, aging between 40-75 years. The sample was non probabilistic type per quota; 30 persons of the (experimental) HRGA and 30 persons of the IHSS (control). The patients were evaluated by personnel of physical culture, Medicine, Infirmary, Psychology, Nutrition and Statistics, during a period of 18 weeks with three weekly follow-ups. There were controlled levels of blood sugar, arterial pressure, pulse, cardiac frequency, saturation of oxygen, weight, and psychological condition. The statistical realized tests were understanding the measures of central trend, dispersion, and T of student. The results indicated: decrease of glucose, hemoglobin glicosilada, of the LDL, triglycerides, total cholesterol, uric acid, corporal mass, index of waist hip and increase of the hemoglobin in the post test. The VO2 Max presented a significant increase, diminishing Rufier's Index. The rapidity of thought and the auto esteem improved in the patients post intervention.

Key words: Diabetes mellitus, physical exercise, life quality. 


\section{INTRODUCCIÓN}

La diabetes es una enfermedad metabólica crónica causada por un defecto heredable, para utilizar carbohidratos, proteínas y grasas secundarias a un déficit relativo 0 absoluto de insulina efectiva, lo que se traduce, en los pacientes no tratados, en un aumento de glucosa en sangre, orina y de lípidos en la sangre. (Augusto, L., 2012). En el tipo (II) que surge en adultos, el cuerpo sí produce insulina, pero, o bien no produce suficiente, o no puede aprovechar la que produce. La insulina no puede escoltar a la glucosa al interior de las células. El tipo (II) suele ocurrir principalmente en personas a partir de los cuarenta años de edad. En la diabetes mellitus tipo (II) 0 no insulinodependiente, no se plantea un factor etiológico asociado a factores tóxicos o infecciosos, no hay relación con antígenos HLA y al parecer, en este tipo de diabetes existe una resistencia a la acción de la insulina, así como una defectuosa secreción de insulina (Chudyk, A. y Petrella, RJ., 2011).

Las complicaciones son frecuentes en el diabético a cualquier edad, y en ocasiones pueden manifestarse desde el inicio clínico de la enfermedad diabética, o tener relación con el tiempo de evolución de la misma. El grado de control metabólico es un factor importante a considerar, ya que en términos generales si el diabético está descompensado (descontrolado), las complicaciones pueden presentarse precozmente, o ser más severas. Como la enfermedad diabética puede afectar todos los sistemas del organismo humano se observan numerosas complicaciones a diferentes niveles (corazón, riñón, vasos periféricos, entre otros). (Chudyk, A. y Petrella, RJ., 2011).

Con respecto a la inclusión del ejercicio en pacientes diabéticos se plantea que: la importancia que tiene en estos pacientes, consiste en que el ejercicio modifica muchos factores de riesgo y disminuye la respuesta inflamatoria en la pared de las arterias (aterosclerosis). (Kwon HR, 2010). El tipo de ejercicio depende de la edad, preparación física, enfermedades asociadas, complicaciones agudas y crónicas, así como preferencias del paciente (conviene que sea aeróbico y de intensidad moderada). El paciente requiere de una valoración previa en busca de complicaciones crónicas que puedan impedir el ejercicio. Los pacientes mayores de 35 años requieren un electrocardiograma previo para descartar la existencia de una cardiopatía isquémica, que limitaría el ejercicio. (Márquez, JJ., Suarez, R. y Márquez, J., 2012).

El ejercicio físico disminuye la glicemia durante y posterior a su realización, mejora la sensibilidad a la insulina, disminuye los lípidos plasmáticos y la tensión arterial, 
provoca reducción del peso y el mantenimiento de la masa corporal delgada, da sensación de bienestar y en general mejora la calidad de vida del diabético. (Kwon $H R, 2010)$. Por todo lo anterior se hicieron las siguientes preguntas de estudio:

¿Cómo mejorar el control metabólico y la calidad de vida de los pacientes diabéticos tipo II del IHSS y el HRGA de la ciudad de Danlí?

¿Qué efectividad posee el programa de ejercicios físicos y educación diabetológica en el control metabólico de los diabéticos tipo II del IHSS de la ciudad de Danlí y en el mejoramiento de la calidad de vida?

Se determinó como objetivo general del trabajo de investigación el evaluar los resultados de la aplicación de un programa de ejercicios físicos y educación diabetológica para mejorar el control metabólico y la calidad de vida de los pacientes diabéticos tipo II del HRGA y el IHSS de la ciudad de Danlí.

La actividad física, sin distingo de edad, género, condición social u ocupación, contiene diferentes beneficios para la salud orgánica, emocional y psíquica de las personas, ya que ofrece herramientas que le permiten al individuo afrontar la vida con una actitud diferente, permite que las personas como entes individuales tengan la vitalidad, vigor, fuerza y energía fundamentales para cumplir con su deber en el grupo social al que pertenecen (Aguilar, Y., Sánchez, V., Llaguno, G., 2012).

En la sección de ciencias médicas y el área de cultura física de UNAH-TEC-Danlí, se ha evidenciado la carencia de programas de ejercicios y orientación educacional de los diabéticos tipo II del IHSS y del Hospital Regional "Gabriela Alvarado". Con este estudio experimental se pretende promover la actividad física con el objeto mejorar la salud, reducir el gasto en compra de medicamentos por el paciente y el Estado, y conocer la patología para poder convivir con ella. El estudio determinó los cambios metabólicos en los que incide la propuesta en mención, referido específicamente a la actividad física y a la educación que se pretende instruir y concientizar antes-durante-después en la aplicación de la intervención en el proceso de la indagación. Entre las implicaciones prácticas y teóricas podemos enunciar que la indagación en curso nos generará un programa de ejercicios y orientación educacional de los diabéticos tipo II adecuado a la población en estudio, el cual podría incorporarse al sistema de salud preventiva de la Secretaría de Salud y el IHSS.

El equipo que se adquirió es utilizado en el gimnasio de actividad física para la salud, sirve para seguir haciendo futuras investigaciones, realizar vinculación con el IHSS y 
el HRGA, desarrollar el proceso docente educativo del área de cultura física y vender servicios a la comunidad a costos menores que en el mercado.

En relación con este criterio de calidad de vida. Define como tratamiento completo de la diabetes "... aquel con el que se logra el control metabólico de la enfermedad..." (Chudyk, A. y Petrella, RJ., 2011). El bienestar de estos enfermos no sólo depende de su control metabólico. Si bien de esta condición depende mucho, dicho bienestar, para lograr el tratamiento debe estar constituido por otros elementos:

- Práctica sistemática de ejercicios físicos adecuadamente planificados y estructurados, teniendo en cuenta las habilidades que se requieren para desarrollar en cada uno de los pacientes.

- Educación Diabetológica, con temas, charlas o el simple diálogo sobre los cuidados, las acciones vinculadas al medicamento, etc. con una superación que, como su nombre lo refiere, este dirigida a un verdadero dominio de los diferentes aspectos que esta área incluye, con la aplicación de métodos pedagógicos, evaluación de los conocimientos, y que responda al diagnóstico individual del paciente y a su nivel de escolaridad e información sobre la enfermedad (American Diabetes Association, 2015).

Es conocido y reportado por múltiples autores que el ejercicio físico aplicado como recurso de la cultura física terapéutica, produce mejorías en las capacidades físicas de estos pacientes e induce a una serie de cambios adaptativos entre los que se incluyen la disminución de la adiposidad, aumento de la capacidad oxidativa metabólica del músculo esquelético, mejoría de las funciones cardiovasculares y respiratoria. A todas estas se agregan: incremento de la capacidad máxima de dioxígeno, normalización de la capacidad de trabajo, incremento de la capacidad vital real, de las que resulta la disminución de la tensión arterial, disminución de la insulina o compuestos orales a consumir en el tratamiento medicamentoso de esta enfermedad (Calles, D., 2011).

El ejercicio físico regular se considera importante en el tratamiento de todos los tipos de diabetes y debe prescribirse por las mismas razones por las que debe fomentarse en la población general (8). Además, la práctica regular de ejercicio físico mejora el control glicémico a largo plazo en los pacientes con diabetes tipo II (no insulinodependiente), mientras que en los pacientes con diabetes tipo I (insulinodependiente), aunque reduce los requerimientos de insulina, no suele modificar el control glicémico. 


\section{Criterios de inclusión}

- Los criterios de inclusión al estudio fueron: Aceptación para la participación en el estudio, residir en la ciudad de Danlí, no tener una morbilidad secundaria a diabetes mellitus (Pre infarto, infarto, angina de pecho, insuficiencia vascular, insuficiencia renal, retinopatía diabética, anemia e hipertensión arterial), no padecer de artritis y artrosis osteoarticulares, no padecer de fibromialgia, edad entre 40-75 años, disponibilidad de horario, dosis de medicamentos.

La muestra: No probabilística por cuota. 30 personas del HRGA. (Grupo experimental) y 30 personas del IHSS. (Grupo control) en base a la capacidad instalada, del medio de transporte disponible y el gimnasio de cultura física.

\section{MÉTODO}

\section{Área geográfica}

El estudio se llevó a cabo en las instalaciones del Gimnasio de Cultura Física y Deportes del Centro Regional UNAH-TEC-DANLÍ, aldea de San Marcos Arriba, carretera panamericana hacia la Ciudad de El Paraíso, de la Ciudad de Danlí, El Paraíso, Honduras.

\section{Definición de caso}

Todo paciente con Diabetes tipo II, edad entre 40-65 años, que acepte participar en el estudio y que no tenga comorbilidad secundaria a la Diabetes mellitus

Los criterios de selección de la muestra para el grupo de control fueron los siguientes: aceptar la participación en el estudio, residir en la ciudad de Danlí, no tener una comorbilidad secundaria a Diabetes mellitus (pre infarto, infarto, angina de pecho, insuficiencia vascular, insuficiencia renal, retinopatía diabética, anemia e hipertensión arterial), no padecer de artritis y artrosis osteoarticulares, no padecer de fibromialgia, tener una edad entre 40-65 años, poseer disponibilidad de horario, y realizar ejercicio físico supervisado.

Los criterios de selección del grupo experimental fueron los siguientes: aceptar la participación en el estudio, residir en la ciudad de Danlí, no tener una comorbilidad 
secundaria a Diabetes mellitus (pre infarto, infarto, angina de pecho, insuficiencia vascular, insuficiencia renal, retinopatía diabética, anemia e hipertensión arterial, no padecer de artritis y artrosis osteoarticulares, no padecer de fibromialgia, tener una edad entre 40-65 años, poseer disponibilidad de horario y no someterse a ejercicio físico supervisado.

Los criterios de exclusión (grupo control y experimental) fueron los siguientes: no aceptar la participación en el estudio, vivir lejos de UNAH TEC Danlí, tener una patología secundaria a la diabetes, ser un paciente con artritis, artrosis osteoarticulares 0 fibromialgia, ser mayor de 65 años, no disponer de horario y no tener una adherencia a medicamentos.

El proceso de selección de personas realizó con una lista de pacientes que padecían de Diabetes mellitus tipo II del Hospital Gabriela Alvarado, del Seguro Social y del grupo de apoyo de pacientes diabéticos que coordinaba el Dr. Mauricio Erazo. También se utilizó una publicidad por radio y televisión con el apoyo del periodista Antonio Gaytan, por familiares y amigos. Con el objetivo de socializar el estudio se realizó una primera reunión en la Alcaldía Municipal de Danlí y las reuniones posteriores para realizar las evaluaciones en las instalaciones del gimnasio UNAH TEC Danlí. Se incluyeron en el estudio de acuerdo a los siguientes criterios utilizados por Ricardo Ibáñez (2009):

En la evaluación médica se evaluó la historia clínica, exámenes físicos (Anamnesis) y exámenes clínicos (Glicemia, Colesterol Total, Triglicéridos, HDL-c, LDL-c, Hemoglobina, Hemoglobina glucosilada y electrocardiograma). En la evaluación en la cultura física se tomó en cuenta el índice de composición corporal (porcentaje de grasa corporal), índice de masa corporal, índice de cintura cadera, índice de Ruffier, test de marcha de 6 minutos (VO2 MAX) y capacidad pulmonar real. En la evaluación psicológica se utilizó la prueba de autoestima, prueba de rapidez de pensamiento y el temperamento. Finalmente en la evaluación instructiva se revisó la prueba de conocimiento de la enfermedad y la prueba de nutrición.

\section{Estimación de la muestra}

La población del IHSS de la ciudad de Danlí comprende 12,432 afiliados directos y 8,503 afiliados indirectos, de los cuales, 2132 de la población en general son diabéticos, 1672 diabéticos tipo II y 460 diabéticos tipo I. La población del HRGA comprende 45,387 pacientes, siendo 28,217 correspondientes a consulta externa, y 17,170 a 
emergencia. 191 de la población general son diabéticos y de estos, 169 son diabéticos tipo II y 22 son del tipo I.

La muestra: No probabilística por cuota. 30 personas del HRGA. (Grupo experimental) y 30 personas del IHSS. (Grupo control) en base a la capacidad instalada, del medio de transporte disponible y el gimnasio de cultura física.

Se describe la Intervención de acuerdo a la siguiente tabla:

\section{Tabla 1.}

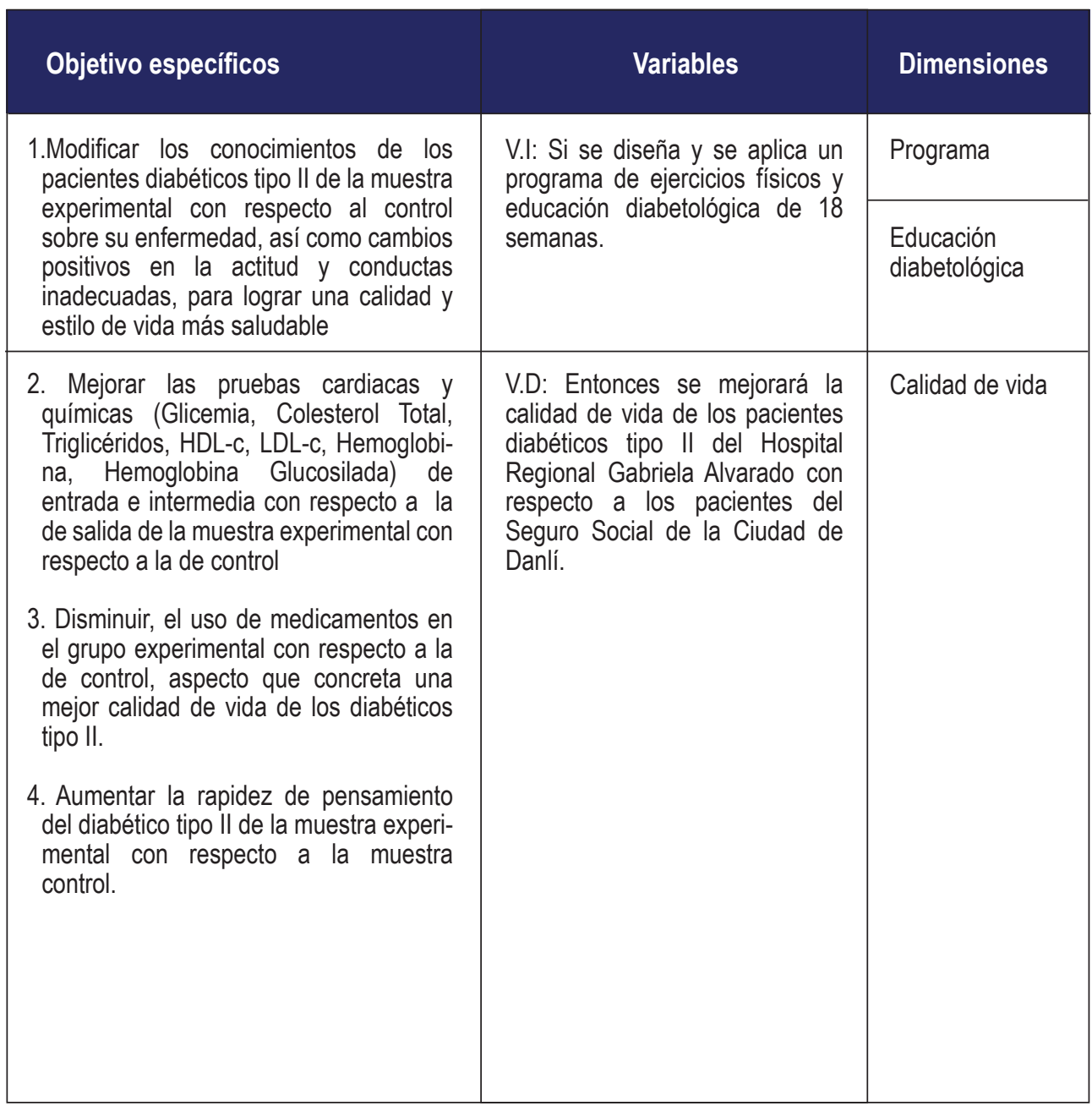




\begin{tabular}{|c|c|c|c|}
\hline Indicadores & Ítems & $\begin{array}{l}\text { Unidades } \\
\text { de análisis }\end{array}$ & Instrumento a utilizar \\
\hline Programa de ejercicio & $\begin{array}{lll}\text { Ver ítems \# } 7 & \text { sobre la } \\
\text { manifestación } & \text { de } & \text { la } \\
\text { Hiperglicemia. } & & \end{array}$ & $\begin{array}{l}60 \\
\text { Personas }\end{array}$ & Cuestionario \\
\hline $\begin{array}{l}\text { Conocimiento de la } \\
\text { enfermedad (Diabetes } \\
\text { Mellitus tipo II) }\end{array}$ & $\begin{array}{l}\text { Ver cuestionario sobre } \\
\text { conocimiento de la } \\
\text { enfermedad } 14 \text { ítems }\end{array}$ & $\begin{array}{l}60 \\
\text { Personas }\end{array}$ & Cuestionario \\
\hline $\begin{array}{l}\text { Conocimiento sobre la } \\
\text { nutrición cuando se } \\
\text { posee la enfermedad } \\
\text { (Diabetes mellitus tipo } \\
\text { II) Ejercicio }\end{array}$ & $\begin{array}{l}\text { Ver cuestionario sobre } \\
\text { conocimiento de nutrición } \\
\text { para convivir con la } \\
\text { enfermedad } 10 \text { ítems }\end{array}$ & $\begin{array}{l}60 \\
\text { Personas }\end{array}$ & Cuestionario \\
\hline $\begin{array}{l}\text { Conocimiento de la } \\
\text { dosificación e importan- } \\
\text { cia de la actividad física } \\
\text { para el tratamiento de } \\
\text { la diabetes mellitus tipo } \\
\text { II. }\end{array}$ & $\begin{array}{l}\text { Ver cuestionario sobre } \\
\text { conocimiento de la impor- } \\
\text { tancia y dosificación del } \\
\text { ejercicio } 7 \text { ítems }\end{array}$ & $\begin{array}{l}60 \\
\text { Personas }\end{array}$ & Cuestionario \\
\hline $\begin{array}{l}\text { Psicológica } \\
\text { Aplicada por la Psicólo- } \\
\text { ga Elena Zúniga. }\end{array}$ & $\begin{array}{l}\text { Ver test de autoestima. } 10 \\
\text { ítems. }\end{array}$ & $\begin{array}{l}60 \\
\text { Personas }\end{array}$ & $\begin{array}{l}\text { - Test de autoestima. } \\
\text { - Test de rapidez de pensa- } \\
\text { miento. } \\
\text { - Test de Temperamento. }\end{array}$ \\
\hline $\begin{array}{l}\text { Fisiológica } \\
\text { Aplicada por los } \\
\text { profesionales en cultura } \\
\text { física, coordinados por } \\
\text { el PhD Raúl Figueroa }\end{array}$ & $\begin{array}{l}\text { Ver test rapidez de pensa- } \\
\text { miento. }\end{array}$ & $\begin{array}{l}60 \\
\text { Personas }\end{array}$ & $\begin{array}{l}\text { - Índice de composición } \\
\text { corporal (Porcentaje de } \\
\text { grasa) } \\
\text { - İndice de masa corporal. } \\
\text { - Índice de cintura cadera. } \\
\text { - Índice de Ruffier. } \\
\text { - Test de marcha de } 6 \\
\text { minutos (VO2 MAX). } \\
\text { - Capacidad pulmonar real. }\end{array}$ \\
\hline $\begin{array}{l}\text { Química } \\
\text { Muestras tomadas por } \\
\text { la las Licenciadas en } \\
\text { Enfermería y elabora- } \\
\text { dos por el Dr. Ismael } \\
\text { Salinas, Microbiólogo } \\
\text { del Hospital Gabriela } \\
\text { Alvarado. }\end{array}$ & Exámenes químicos & $\begin{array}{l}60 \\
\text { Personas }\end{array}$ & $\begin{array}{l}\text { - Historia clínica. } \\
\text { Elaborada por el Médico } \\
\text { Internista Daniel Alvarado. } \\
\text { - Exámenes físicos } \\
\text { - Examen cardíaco. } \\
\text { - Exámenes clínicos (Glice- } \\
\text { mia, Colesterol Total, } \\
\text { Triglicéridos, HDL-c, LDL-c, } \\
\text { Hemoglobina, Hemoglobi- } \\
\text { na Glucosilada }\end{array}$ \\
\hline
\end{tabular}

Todas las evaluaciones psicológicas, clínicas, laboratoriales y físicas, se hicieron en tres momentos: al inicio para la captación de la muestra, a la novena semana (intermedia) y al final del programa implementado. Durante la implementación del programa, diariamente se monitoreaba: frecuencia cardíaca, frecuencia respiratoria, glucometría y presión arterial.

Fuente propia 


\section{Pruebas estadísticas realizadas en ambos grupos de estudio:}

El estudio tiene un enfoque cuantitativo y cuasi experimental. Para ello se estudiaron dos grupos: experimental (experimento-intervención) y grupo de control. La muestra utilizada fue no probabilística por cuota: 30 personas del HRGA. (Grupo experimental) y 30 personas del IHSS. (Grupo control) en base a la capacidad instalada del medio de transporte disponible y el gimnasio de cultura física.

Las pruebas estadísticas utilizadas fueron: medidas de tendencia central, de dispersión y la prueba de significancia fue $T$ de student (correlación de variables). Los programas informáticos utilizados fueron: el Excel y el Statistical Analisis Sistem (SAS). Los casos fueron evaluados por personal del departamento de cultura física y deportes y equipo médico, Enfermería, Psicología, Nutricionista y Estadístico. Durante 18 semanas, en tres seguimientos por semana, se controlaba niveles de glicemia, presión arterial, pulso, frecuencia cardiaca, niveles de saturación de oxígeno, peso y estado psicológico del grupo experimental y control.

\section{ANÁLISIS}

Instrumentos y técnicas de investigación. Mediciones a realizar a los diabéticos tipo II.

Índice de composición corporal:

El porcentaje de grasa corporal se determina por medio de la siguiente ecuación: (Parizkova y Buzcova, 1971). El porcentaje de grasa corporal no es otra cosa que la cantidad de grasa en relación al peso total de tu cuerpo.

$\% G=2.745+0.0008\left(X_{1}\right)+0.002\left(X_{2}\right)+0.637\left(X_{3}\right)+0.809\left(X_{4}\right)$

Dónde: \% G = Porcentaje de grasa corporal.

1. $(X 1)$ = Pliegue cutáneo tricipital $(\mathrm{mm})$

2. $(\mathrm{X} 2)$ = Pliegue cutáneo infra-escapular $(\mathrm{mm})$

3. $(\mathrm{X} 3)=$ Pliegue cutáneo supra-iliaco $(\mathrm{mm})$

4. $(X 4)=$ Pliegue cutáneo bicipital $(\mathrm{mm})$ 
Imágenes 1, 2,3 y 4.
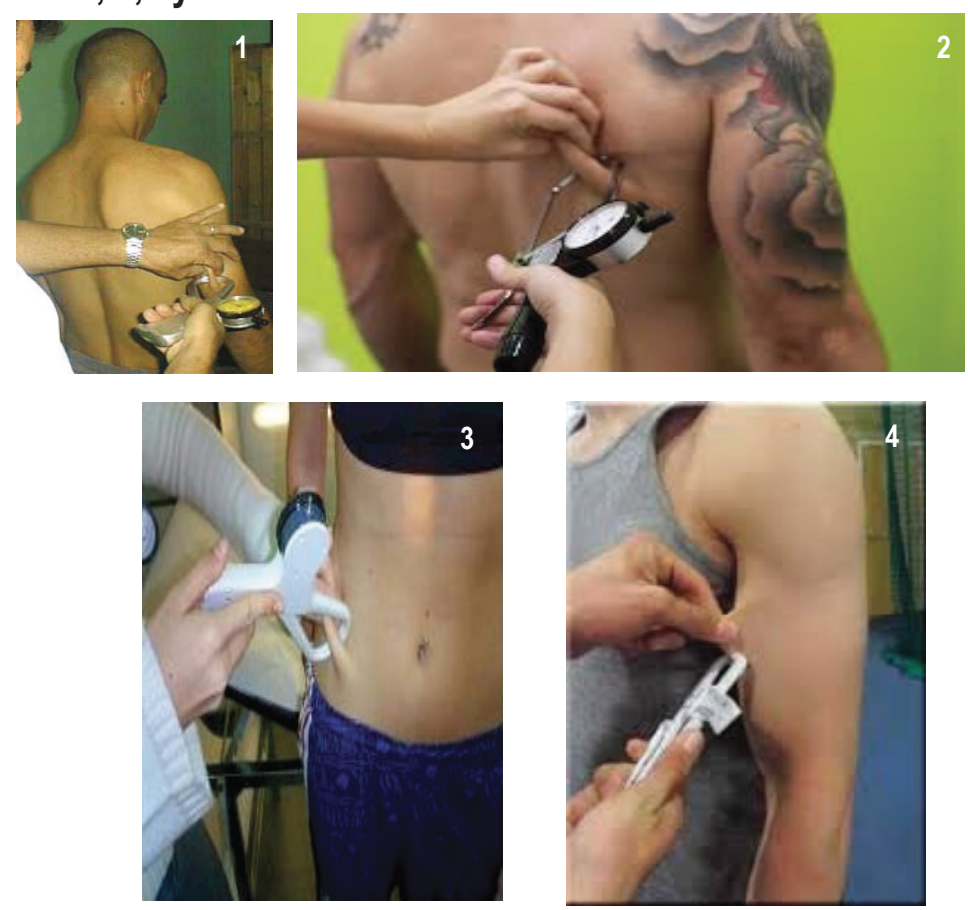

Tabla 2.

\begin{tabular}{|l|l|l|l|l|l|}
\hline Mujer & \multicolumn{5}{|c|}{ DATOS EN PORCENTAJE } \\
\hline Edad & Excelente & Buena & Normal & $\begin{array}{l}\text { Sobrepes } \\
0\end{array}$ & Obesidad \\
\hline Menor o igual 19 & 17.00 & $17.1-22.0$ & $22.1-27$ & $27.1-32.0$ & Mayor igual 32.1 \\
\hline $\mathbf{2 0 - 2 9}$ & 18.00 & $18.1-23$ & $23.1-28$ & $28.1-33.0$ & Mayor igual 33.1 \\
\hline $\mathbf{3 0 - 3 9}$ & 19.00 & $19.1-24$ & $24.1-29$ & $29.1-34.0$ & Mayor igual 34.1 \\
\hline $\mathbf{4 0 - 4 9}$ & 20.00 & $20.1-25$ & $25.1-30$ & $30.1-35.0$ & Mayor igual 35.1 \\
\hline Mayor o igual 50 & 21.00 & $21.1-26$ & $26.2-31.0$ & $31.1-36.0$ & Mayor igual 36.1 \\
\hline \multicolumn{5}{|l|}{ DATOS EN PORCENTAJE } \\
\hline Hombre & \multicolumn{5}{|l|}{} \\
\hline Edad & Excelente & Buena & Normal & $\begin{array}{l}\text { Sobrepes } \\
0\end{array}$ & Obesidad \\
\hline Menor igual 19 & 12.0 & $12.1-17.0$ & $17.1-22.0$ & $22.1-27.0$ & Mayor igual 271 \\
\hline $\mathbf{2 0 - 2 9}$ & 13.0 & $13.1-18.0$ & $18.1-23.0$ & $23.1-28.0$ & Mayor igual 28.1 \\
\hline $\mathbf{3 0 - 3 9}$ & 14.0 & $14.1-19.0$ & $19.1-24.0$ & $24.1-29.0$ & Mayor igual 29.1 \\
\hline $\mathbf{4 0 - 4 9}$ & 15.0 & $15.1-20-0$ & $20.1-25.0$ & $25.1-30.0$ & Mayor igual 30.1 \\
\hline Mayor o igual 50 & 16.0 & $16.1-21.0$ & $21.1-26.0$ & $26.1-31.0$ & Mayor igual 31.1 \\
\hline
\end{tabular}

Fuente: Norton 1996. 
Tabla 3. Porcentaje de grasa corporal en base a la suma de 4 pliegues subcutáneos (bíceps, tríceps, subescapular y supra iliaco para ambos sexos).

\begin{tabular}{|c|c|c|c|c|c|c|c|c|}
\hline \multirow[t]{2}{*}{$\Sigma 4$ Pliegues } & \multicolumn{4}{|c|}{ Hombres (edad en años) } & \multicolumn{4}{|c|}{ Mujeres (edad en años) } \\
\hline & $17-29$ & $30-39$ & $40-49$ & $50+$ & $16-29$ & $30-39$ & $40-49$ & $50+$ \\
\hline 15 & 4.8 & & & & 10.5 & & & \\
\hline 20 & 8.1 & 12.2 & 12.2 & 12.6 & 14.1 & 17.0 & 19.8 & 21.4 \\
\hline 25 & 10.5 & 14.2 & 15.0 & 15.6 & 16.8 & 19.4 & 22.2 & 24.0 \\
\hline 30 & 12.9 & 16.2 & 17.7 & 18.6 & 19.5 & 21.8 & 24.5 & 26.6 \\
\hline 35 & 14.7 & 17.7 & 19.6 & 20.8 & 21.5 & 23.7 & 26.4 & 28.5 \\
\hline 40 & 16.4 & 19.2 & 21.4 & 22.9 & 23.4 & 25.5 & 28.2 & 30.3 \\
\hline 45 & 17.7 & 20.4 & 23.0 & 24.7 & 25.0 & 26.9 & 29.6 & 31.9 \\
\hline 50 & 19.0 & 21.5 & 24.6 & 26.5 & 26.5 & 28.2 & 31 & 33.4 \\
\hline 55 & 20.1 & 22.5 & 25.9 & 27.9 & 27.8 & 29.4 & 32.1 & 346 \\
\hline 60 & 21.2 & 23.5 & 27.1 & 29.2 & 29.1 & 30.6 & 33.2 & 35.7 \\
\hline 65 & 22.2 & 24.3 & 28.2 & 30.4 & 30.2 & 31.6 & 34.1 & 36.7 \\
\hline 70 & 23.1 & 25.1 & 29.3 & 31.6 & 31.2 & 32.5 & 35.0 & 37.7 \\
\hline 75 & 24.0 & 25.9 & 30.3 & 32.7 & 32.2 & 33.4 & 35.9 & 38.7 \\
\hline 80 & 24.8 & 26.6 & 31.2 & 33.8 & 33.1 & 34.3 & 36.7 & 39.6 \\
\hline 85 & 25.5 & 27.2 & 32.1 & 34.8 & 34.0 & 35.1 & 37.5 & 40.4 \\
\hline 90 & 26.2 & 27.8 & 33.0 & 35.8 & 34.8 & 35.8 & 38.3 & 41.2 \\
\hline 95 & 26.9 & 28.4 & 33.7 & 36.6 & 35.6 & 36.5 & 39.0 & 41.9 \\
\hline 100 & 27.6 & 29.0 & 34.4 & 37.4 & 36.4 & 37.2 & 39.7 & 42.6 \\
\hline 105 & 28.2 & 29.6 & 35.1 & 38.2 & 37.1 & 379 & 40.4 & 43.8 \\
\hline 110 & 28.8 & 30.1 & 35.8 & 39.0 & 37.8 & 38.6 & 41.0 & 43.9 \\
\hline 115 & 29.4 & 30.6 & 36.4 & 39.7 & 38.4 & 39.1 & 41.5 & 44.5 \\
\hline 120 & 30.0 & 31.1 & 37.0 & 40.4 & 39.0 & 39.6 & 42.0 & 45.1 \\
\hline 125 & 31.0 & 31.5 & 37.6 & 41.1 & 39.6 & 40.1 & 42.5 & 45.7 \\
\hline 130 & 31.5 & 31.9 & 38.2 & 41.8 & 40.2 & 40.6 & 43.0 & 46.2 \\
\hline 135 & 32.0 & 32.3 & 38.7 & 42.4 & 40.8 & 41.1 & 43.5 & 46.9 \\
\hline 140 & 32.5 & 32.7 & 39.2 & 43.0 & 41.3 & 41.6 & 44.0 & 47.2 \\
\hline 145 & 32.9 & 33.1 & 39.7 & 43.6 & 41.8 & 42.1 & 44.5 & 47.7 \\
\hline 150 & 33.3 & 33.5 & 40.2 & 44.1 & 42.3 & 42.6 & 45.0 & 48.2 \\
\hline 155 & 33.7 & 33.9 & 40.7 & 44.6 & 423 & 43.1 & 45.4 & 48.7 \\
\hline 160 & 34.1 & 34.3 & 41.2 & 45.1 & 42.8 & 43.6 & 45.8 & 49.2 \\
\hline 165 & 34.5 & 34.6 & 41.6 & 45.6 & 43.3 & 44.0 & 46.2 & 49.6 \\
\hline 170 & 34.9 & 34.8 & 42 & 46.1 & 43.7 & 44.4 & 46.6 & 50.0 \\
\hline 175 & 353 &. & . & . & 44.1 & 44.8 & 47.0 & 50.4 \\
\hline 180 & 35.6 &. & - & . &. & 45.2 & 47.4 & 50.8 \\
\hline 185 & 35.9 &. & - &. & - & 45.6 & 47.8 & 51.2 \\
\hline 190 &. & . & 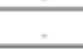 &. & 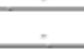 & 45.9 & 48.2 & 51.6 \\
\hline 195 & & & & & & 46.2 & 48.5 & 520 \\
\hline
\end{tabular}

Fuente:

- Withers RT, Whittingham NO, Norton K1 et al Relative body fat and anthropometric prediction of body density of famales athletes. Eur. J Appl Physiol 1987; 56:169-180. 
Índice de masa corporal

Determina la masa adecuada de los sujetos y su grado de obesidad si la hay.

Su objetivo radica en: Valorar la aceptabilidad o línea de "normalidad" en la relación a la masa o peso y la estatura, el sobrepeso y la obesidad, así como percibir ciertos grados de mal nutrición.

IMC = M (Kg.)/ (Talla m. $)^{2}$

Donde:

IMC = Índice de masa corporal

$M=$ Masa o peso en kilogramos.

Tabla 4. Clasificación del estado nutricional de acuerdo con el índice de masa corporal

\begin{tabular}{|l|c|c|}
\hline \multicolumn{1}{|c|}{ Clasificación } & IMC $\left(\mathbf{K g} \cdot \mathrm{m}^{2}\right)$ & Riesgo de morbilidad \\
\hline Bajo peso & $<18,5$ & Bajo (c/ riesgos de otros problemas clinicos) \\
\hline Normal & $18,5-24,9$ & Promedio \\
\hline Sobrepeso & $\geq 25,0$ & \\
\hline Preobeso & $25,0-29,9$ & Incrementado \\
\hline Obeso Grado I & $30,0-34,9$ & Moderado \\
\hline Obeso Grado II & $35,0-39,9$ & Severo \\
\hline Obeso Grado III & $\geq 40,0$ & Muy severo \\
\hline
\end{tabular}

Fuente:

- OMS. Obesity. Preventing and managing the global epidemic. Report of a WHO Consulting on Obesity. Geneva: WHONUT/NCD $198.1,1998 ; 9$

\section{Índice de cintura cadera.}

Objetivo: Estima el riesgo de padecer enfermedades cardiovasculares en personas sedentarias.

$\mathrm{CC}=$ Circunferencia de cintura o abdominal

Circunferencia de cadera 


\section{Tabla 5.}

\begin{tabular}{|c|c|c|c|c|c|c|c|c|}
\cline { 2 - 9 } \multicolumn{1}{c|}{} & \multicolumn{6}{c|}{ NIVLES DE RIESGO NDICE CINTURA CADAERA } \\
\cline { 2 - 10 } \multicolumn{1}{c|}{} & \multicolumn{2}{c|}{ Muy Alto } & \multicolumn{2}{c|}{ Alto } & \multicolumn{2}{c|}{ Moderado } & \multicolumn{2}{c|}{ Bajo } \\
\hline $\begin{array}{l}\text { Grupes } \\
\text { Etarios }\end{array}$ & H & II & H & M & H & M & H & II \\
\hline 20 a 29 & $>0.94$ & $>0.82$ & 0.88 a 0.94 & 0.77 a 0.82 & 0.83 a 0.87 & 0.71 a 0.76 & $<0.83$ & $<0.71$ \\
\hline 30 a 39 & $>0.96$ & $>0.85$ & 0.92 a 0.96 & 0.78 a 0.85 & 0.84 a 0.91 & 0.72 a 0.77 & $<0.84$ & $<0.72$ \\
\hline 40 a 49 & $>1.01$ & $>0.87$ & 0.95 a 1.01 & 0.80 a 0.87 & 0.88 a 0.94 & 0.73 a 0.79 & $<0.88$ & $<0.73$ \\
\hline 50 a 59 & $>1.02$ & $>0.88$ & 0.96 a 1.02 & 0.82 a 0.88 & 0.900 .95 & 0.74 a 0.81 & $<0.9$ & $<0.74$ \\
\hline 60 a 69 & $>1.03$ & $>0.91$ & 0.98 a 1.03 & 0.85 a 0.91 & 0.91 a 0.97 & 0.76 a 0.84 & $<0.91$ & $<0.76$ \\
\hline
\end{tabular}

Fuente:

- De Valera Hernández, Yolanda. Manual para Simplificar la Evaluación Nutricional Antropométrica en Adultos. U.S.B. 1995.

Índice de masa corporal

Test de Ruffier (12):

Objetivo: Medir la adaptación cardiovascular al esfuerzo.

Metodología:

- Tomar pulso en reposo (P).

- Aplicar carga (30 cuclillas en 45 seg.).

- Tomar pulso inmediatamente finalizada la carga (P1).

- Tomar pulso pasado un minuto luego de terminada la carga (P2).

- Aplicar la siguiente fórmula:

$$
R=\frac{(P+P 1+P 2-200)}{10}
$$

\section{Tabla 6. Parámetros de valoración}

\begin{tabular}{|l|l|}
\hline Excelente & Menor que 0 \\
\hline Bien & $1-5$ \\
\hline Regular & $6-10$ \\
\hline Pobre & $11-15$ \\
\hline Malo & Mayor que 16 \\
\hline
\end{tabular}


Test de caminata de los 6 minutos

Objetivo: Evaluar de forma sistemática o periódica la capacidad funcional a los pacientes que participan en los programas de ejercicios físicos.

\section{Metodología de la prueba}

- Mantener en reposo durante 10 minutos al paciente antes de comenzar la prueba.

- Realizar tomas de frecuencia cardíaca en reposo, al culminar la prueba y pasado el primer minuto de recuperación.

- Realizar tomas de presión arterial en reposo, al culminar la prueba y pasado el primer minuto de recuperación.

- Explicarle detalladamente la prueba al paciente antes de comenzar la misma.

- Caminar al máximo de las posibilidades de cada paciente durante 6 minutos.

- Medir la distancia recorrida en metros.

Indicadores fisiológicos que se obtienen a partir del resultado del test de los 6 minutos.

- Consumo máximo de oxigeno (VO2 máx.).

Fórmulas para determinar el VO2 máx.

El máximo consumo de oxígeno se obtiene por la fórmula propuesta por el "Colegio Americano de Medicina del Deporte" (CAMD.), para aquellos pacientes que caminaban con una intensidad entre 50 y 100 metros por minutos, para los que lo hicieran con una intensidad entre 100 y 130 metros por minuto se utiliza la fórmula del Colegio Americano de Medicina del Deporte modificada por el equipo de trabajo del Centro Actividad Física y Salud del ISCF "Manuel Fajardo".

Fórmula del (CAMD.): Modificada.

$\mathrm{VO} 2=[(\mathrm{mts} / \mathrm{t})-133] * 0.17+33=$

VO2 $=[(920 / 6)-133]^{*} 0.17+33=36.46 \mathrm{ml} / \mathrm{kg} / \mathrm{min}$. (Este es un ejemplo). 
Tabla 7. Evaluación para el Consumo Máximo de Oxigeno según V.M. Zatsiorki.

\begin{tabular}{|l|l|l|l|l|}
\hline 1. & Si el CO está entre & 53.4 y $60.1 \mathrm{ml} / \mathrm{kg} / \mathrm{min}$ & el nivel será & Muy bueno \\
\hline 2. & Si el CO está entre & 46.0 y $53.3 \mathrm{ml} / \mathrm{kg} / \mathrm{min}$ & el nivel será & Bueno \\
\hline 3. & Si el CO e stá entre & 39.8 y $45.9 \mathrm{ml} / \mathrm{kg} / \mathrm{min}$ & el nivel será & Regular \\
\hline 4. & Si el CO está entre & 36.4 y $39.7 \mathrm{ml} / \mathrm{kg} / \mathrm{min}$ & el nivel será & Pobre \\
\hline 5. & Si el CO es menor de & $36.3 \mathrm{ml} / \mathrm{kg} / \mathrm{min}$ & el nivel será & Muy pobre \\
\hline
\end{tabular}

Capacidad real pulmonar

Se refieren a los distintos volúmenes de aire característicos en la respiración humana. Un pulmón humano puede almacenar alrededor de 5 litros de aire en su interior, pero una cantidad significativamente menor es la que se inhala y exhala durante la respiración.

\section{Tabla 8. Nomograma Predictivo - Capacidad Inspiratoria}

\begin{tabular}{|c|c|c|c|c|c|c|c|c|c|c|}
\hline & \multicolumn{9}{|c|}{ Altura } \\
\hline & & $58 "$ & $60 "$ & $62 "$ & $64 "$ & $66 "$ & $68^{\prime \prime}$ & $70 "$ & 72" & $74^{\prime \prime}$ \\
\hline & & $1.47 \mathrm{~m}$ & $1.52 \mathrm{~m}$ & $1.57 \mathrm{~m}$ & $1.63 \mathrm{~m}$ & $1.68 \mathrm{~m}$ & $1.73 \mathrm{~m}$ & $1.78 \mathrm{~m}$ & $1.83 \mathrm{~m}$ & $1.88 \mathrm{~m}$ \\
\hline \multirow{13}{*}{ Mujer } & 20 & 1900 & 2100 & 2300 & 2500 & 2700 & 2900 & 3100 & 3300 & 3500 \\
\hline & 25 & 1850 & 2050 & 2250 & 2450 & 2650 & 2850 & 3050 & 3250 & 3450 \\
\hline & 30 & 1800 & 2000 & 2200 & 2400 & 2600 & 2800 & 3000 & 3200 & 3400 \\
\hline & 35 & 1750 & 1950 & 2150 & 2350 & 2550 & 2750 & 2950 & 3150 & 3350 \\
\hline & 40 & 1700 & 1900 & 2100 & 2300 & 2500 & 2700 & 2900 & 3100 & 3300 \\
\hline & 45 & 1650 & 1850 & 2050 & 2250 & 2450 & 2650 & 2850 & 3050 & 3250 \\
\hline & 50 & 1600 & 1800 & 2000 & 2200 & 2400 & 2600 & 2800 & 3000 & 3200 \\
\hline & 55 & 1550 & 1750 & 1950 & 2150 & 2350 & 2550 & 2750 & 2950 & 3150 \\
\hline & 60 & 1500 & 1700 & 1900 & 2100 & 2300 & 2500 & 2700 & 2900 & 3100 \\
\hline & 65 & 1450 & 1650 & 1850 & 2050 & 2250 & 2450 & 2650 & 2850 & 3050 \\
\hline & 70 & 1400 & 1600 & 1800 & 2000 & 2200 & 2400 & 2600 & 2800 & 3000 \\
\hline & 75 & 1350 & 1550 & 1750 & 1950 & 2150 & 2350 & 2550 & 2750 & 2950 \\
\hline & 80 & 1300 & 1500 & 1700 & 1900 & 2100 & 2300 & 2500 & 2700 & 2900 \\
\hline
\end{tabular}

\begin{tabular}{|c|c|c|c|c|c|c|c|c|c|c|c|c|}
\hline \multirow{2}{*}{\multicolumn{2}{|c|}{ 吾 }} & \multicolumn{11}{|c|}{ Altura } \\
\hline & & $\begin{array}{c}58 " \\
1.47 \mathrm{~m}\end{array}$ & $\begin{array}{c}60 " \\
1.52 \mathrm{~m}\end{array}$ & $\begin{array}{c}62 " \\
1.57 \mathrm{~m}\end{array}$ & $\begin{array}{c}64 " \\
1.63 \mathrm{~m}\end{array}$ & $\begin{array}{c}66 " \\
1.68 \mathrm{~m}\end{array}$ & $\begin{array}{c}688^{\prime \prime} \\
1.73 \mathrm{~m}\end{array}$ & \begin{tabular}{|c|}
$70 "$ \\
$1.78 \mathrm{~m}$
\end{tabular} & $\begin{array}{c}72^{\prime \prime} \\
1.83 \mathrm{~m}\end{array}$ & $\begin{array}{c}74^{\prime \prime} \\
1.88 \mathrm{~m}\end{array}$ & $\begin{array}{c}76^{\prime \prime} \\
1.93 \mathrm{~m}\end{array}$ & $\begin{array}{c}78^{\prime \prime} \\
1.98 \mathrm{~m}\end{array}$ \\
\hline \multirow{13}{*}{ Hombre } & 20 & 2000 & 2200 & 2400 & 2600 & 2800 & 3000 & 3200 & 3400 & 3600 & 3800 & 4000 \\
\hline & 25 & 1950 & 2150 & 2350 & 2550 & 2750 & 2950 & 3150 & 3350 & 3550 & 3750 & 3950 \\
\hline & 30 & 1900 & 2100 & 2300 & 2500 & 2700 & 2900 & 3100 & 3300 & 3500 & 3700 & 3900 \\
\hline & 35 & 1800 & 2000 & 2200 & 2400 & 2600 & 2800 & 3000 & 3200 & 3400 & 3600 & 3800 \\
\hline & 40 & 1750 & 1950 & 2150 & 2350 & 2550 & 2750 & 2950 & 3150 & 3350 & 3550 & 3750 \\
\hline & 45 & 1700 & 1900 & 2100 & 2300 & 2500 & 2700 & 2900 & 3100 & 3300 & 3500 & 3700 \\
\hline & 50 & 1650 & 1850 & 2050 & 2250 & 2450 & 2650 & 2850 & 3050 & 3250 & 3450 & 3650 \\
\hline & 55 & 1550 & 1750 & 1950 & 2200 & 2350 & 2550 & 2750 & 2950 & 3150 & 3350 & 3550 \\
\hline & 60 & 1500 & 1700 & 1900 & 2100 & 2300 & 2500 & 2700 & 2900 & 3100 & 3300 & 3500 \\
\hline & 65 & 1400 & 1600 & 1800 & 2000 & 2200 & 2400 & 2600 & 2800 & 3000 & 3200 & 3400 \\
\hline & 70 & 1350 & 1550 & 1750 & 1950 & 2150 & 2350 & 2550 & 2750 & 2950 & 3150 & 3350 \\
\hline & 75 & 1300 & 1500 & 1700 & 1900 & 2100 & 2300 & 2500 & 2700 & 2900 & 3100 & 3300 \\
\hline & 80 & 1250 & 1450 & 1650 & 1850 & 2050 & 2250 & 2450 & 2650 & 2850 & 3050 & 3250 \\
\hline
\end{tabular}

Fuente propia 


\section{RESULTADOS}

Cuadro 1. Glucosa, hemoglobina glucosilada, ácido úrico y hemoglobina en hombres, mujeres e integrado; antes y a las 18 semanas de la aplicación del programa de ejercicio físico y educación diabetológica.

Hombres

\begin{tabular}{|l|c|c|c|c|}
\hline & Glucosa & Hemog. Glucociliada & Ácido Urico & Hemoglobina \\
\hline Sujeto de estudio & 9 & 9 & 9 & 9 \\
Pre test & $238.1 \pm 87.2$ & $7.6 \pm 1.7$ & $5.3 \pm 1.6$ & $14.0 \pm 1.3$ \\
Post test & $100.1 \pm 13.6$ & $5.8 \pm 0.38$ & $3.6 \pm 073$ & $14.2 \pm 0.70$ \\
Diferencia & $138.0 \pm 62.4$ & $1.86 \pm 1.24$ & $1.7 \pm 1.3$ & $0.19 \pm 1.04$ \\
Probabilidad & 0.0014 & 0.0117 & 0.0155 & 0.7058 \\
\hline
\end{tabular}

Mujeres

\begin{tabular}{|l|c|c|c|c|}
\hline & Glucosa & Hemog. Glucociliada & Ácido Urico & Hemoglobina \\
\hline Sujeto de estudio & 21 & 21 & 21 & 21 \\
Pre test & $221.5 \pm 109.5$ & $8.2 \pm 1.0$ & $4.7 \pm 2.0$ & $12.5 \pm 1.2$ \\
Post test & $100.4 \pm 12.3$ & $5.7 \pm 0.41$ & $3.8 \pm 1.0$ & $12.8 \pm 0.82$ \\
Diferencia & $121.1 \pm 77.9$ & $2.5 \pm 0.80$ & $0.88 \pm 1.60$ & $0.33 \pm 1.01$ \\
Probabilidad & 0.0001 & 0.0001 & 0.0856 & 0.2972 \\
\hline
\end{tabular}

Hombres y Mujeres

\begin{tabular}{|l|c|c|c|c|}
\hline & Glucosa & Hemog. Glucociliada & Ácido Urico & Hemoglobina \\
\hline Sujeto de estudio & 30 & 30 & 30 & 30 \\
Pre test & $226.5 \pm 102.1$ & $8.0 \pm 1.3$ & $4.9 \pm 1.9$ & $12.9 \pm 1.4$ \\
Post test & $100.3 \pm 12.4$ & $5.7 \pm 0.40$ & $3.8 \pm 0.92$ & $13.2 \pm 0.99$ \\
Diferencia & $126.2 \pm 72.72$ & $2.3 \pm 0.95$ & $1.1 \pm 1.5$ & $0.29 \pm 1.2$ \\
\hline Probabilidad & 0.0001 & 0.0001 & 0.0059 & 0.3579 \\
\hline
\end{tabular}

Fuente propia

La glucosa, hemoglobina glucosilada ácido úrico y hemoglobina, se midieron en los hombres, se encontraron diferencias significativas $(P=0.0014, P=0.0117$ y $P=0.0155)$, sin embargo, la hemoglobina normal no presentó un cambio significativo ( $P=0.7058)$. La glucosa y hemoglobina glucosilada se midieron en las mujeres, se encontraron diferencias significativas ( $\mathrm{P}=0.0001$ y $\mathrm{P}=0.0001)$, no obstante, en el ácido úrico no es significativo, pero posee una tendencia fuerte a disminuir, con una $P=0.0856$ y la hemoglobina normal no es significativa, porque muestra una probabilidad después de 18 semanas de $P=0.2972$. Lo que significa que en atención al ácido úrico que 11 de cada 12 personas de género femenino que se someten al programa reducen ese aspecto. 
La glucosa, hemoglobina glucosilada y ácido úrico, se midieron en los hombres y mujeres, se encontraron diferencias significativas $P=0.0001, P=0.0001$ y $P=0.0059$. Sin embargo, la hemoglobina normal no presentó un cambio significativo $(P=0.3579)$, esto demuestra que dos de cada tres personas que se someten al programa de ejercicio mejora su hemoglobina.

Cuadro 2. Triglicéridos, colesterol, colesterol bueno (HDL) y colesterol malo (LDL) y la relación LDL/HDL en hombres, mujeres e integrado; antes y a las 18 semanas de la aplicación del programa de ejercicio físico y educación diabetológica.

Hombres

\begin{tabular}{|l|c|c|c|c|c|}
\hline & Triglicéridos & Colesterol & HDL & LDL & Relación LDL / HDL \\
\hline Sujeto de estudio & 9 & 9 & 9 & 9 & 9 \\
Pre test & $176.9 \pm 61.1$ & $205.5 \pm 30.6$ & $42.0 \pm 13.7$ & $122.8 \pm 36.3$ & 2.9 \\
Post test & $113.8 \pm 32.0$ & $155.3 \pm 21.1$ & $22.9 \pm 3.3$ & $113.7 \pm 18.4$ & 4.97 \\
Diferencia & $63.1 \pm 48.8$ & $50.2 \pm 26.3$ & $19.1 \pm 10.0$ & $9.2 \pm 28.8$ & 2.04 \\
\hline Probabilidad & 0.0014 & 0.0009 & 0.0028 & 0.5085 & 0.0018 \\
\hline
\end{tabular}

Mujeres

\begin{tabular}{|l|c|c|c|c|c|}
\hline & Triglicéridos & Colesterol & HDL & LDL & Relación LDL / HDL \\
\hline Sujeto de estudio & 21 & 21 & 21 & 21 & 21 \\
Pre test & $194.5 \pm 110.7$ & $200.7 \pm 41.5$ & $51.4 \pm 14.4$ & $116.7 \pm 36.4$ & 2.3 \\
Post test & $113.1 \pm 26.1$ & $153.3 \pm 25$ & $26.4 \pm 3.6$ & $112.1 \pm 22.7$ & 4.2 \\
Diferencia & $81.3 \pm 80.4$ & $47.4 \pm 34.3$ & $25.0 \pm 10.5$ & $4.6 \pm 30.3$ & 6.4 \\
\hline Probabilidad & 0.0034 & 0.0001 & 0.0001 & 0.6266 & 0.0001 \\
\hline
\end{tabular}

Hombres y Mujeres

\begin{tabular}{|l|c|c|c|c|c|}
\hline & Triglicéridos & Colesterol & HDL & LDL & Relación LDL / HDL \\
\hline Sujeto de estudio & 30 & 30 & 30 & 30 & 30 \\
\hline Pre test & $189.2 \pm 97.8$ & $202.1 \pm 38.1$ & $51.4 \pm 14.4$ & $118.6 \pm 35.8$ & 2.4 \\
Post test & $113.3 \pm 27.4$ & $153.9 \pm 23.5$ & $26.4 \pm 3.6$ & $112.6 \pm 21.2$ & 5.3 \\
Diferencia & $75.9 \pm 71.8$ & $48.2 \pm 31.7$ & $23.0 \pm 10.5$ & $6 \pm 29.4$ & 3.9 \\
\hline Probabilidad & 0.0034 & 0.0001 & 0.0001 & 0.6266 & 0.0001 \\
\hline
\end{tabular}

Fuente propia

Los triglicéridos, el colesterol y el colesterol bueno o HDL, se midieron en los hombres, se encontraron diferencias significativas $(P=0.0144, P=0.0009$ y $P=0.0028)$, sin embargo, el colesterol malo (LDL) no presentó un cambio significativo $(P=0.5085)$. Demostrando que de dos de tres personas que se someten al programa de ejercicio físico mejoran dicho componente. Cabe señalar que la relación LDL, o sea, colesterol malo con respecto al HDL o colesterol, es altamente significativo, lo que significa que 
los varones que realizan ejercicio físico reducen el riesgo coronario.

Los triglicéridos, el colesterol y el colesterol bueno o HDL se midieron en las mujeres, se encontraron diferencias significativas $(P=0.0034, P=0.0001$ y $P=0.0001)$, sin embargo, el colesterol malo (LDL) no presentó un cambio significativo $(P=0.6266)$, demostrando que una de dos personas que se someten al programa de ejercicio físico mejoran dicho componente. Cabe aclarar que la relación LDL, es decir, colesterol malo con respecto al HDL posee una tendencia débil. Lo que significa que las mujeres que realizan ejercicio físico reducen el riesgo coronario.

Los triglicéridos, el colesterol y el colesterol bueno o HDL se midieron en los hombres y mujeres, se encontraron diferencias significativas $(P=0.0003, P=0.0001$ y $\mathrm{P}=0.0001$ ), pero, el colesterol malo o LDL no presentó un cambio significativo ( $\mathrm{P}=$ 0.4364 ). Sin embargo, esto demuestra que una de dos personas mejoró, lo que indica que el $50 \%$ de los que se sometieron al ejercicio redujeron el riesgo coronario.

Cuadro 3. Masa $\mathrm{kg}$, talla $\mathrm{cm}$, porcentaje de grasa, índice de masa corporal e índice cintura cadera en hombres, mujeres e integrado; antes y a las 18 semanas de la aplicación del programa de ejercicio físico y educación diabetológica.

\begin{tabular}{|l|c|c|c|c|c|}
\hline \multicolumn{1}{|c}{} & Masa Kg & Talla cm & $\begin{array}{c}\text { Porcent. } \\
\text { Grasa }\end{array}$ & IMC & $\begin{array}{c}\text { Ind. Cintura } \\
\text { Cadera }\end{array}$ \\
\hline Sujeto de estudio & 9 & 9 & 9 & 9 & 9 \\
Pre test & $78.4 \pm 16.4$ & $164.6 \pm 4.7$ & $29.8 \pm 5.8$ & $28.6 \pm 5.5$ & $0.98 \pm 0.14$ \\
Post test & $74.2 \pm 14.4$ & $164.6 \pm 4.7$ & $27.4 \pm 4.4$ & $27.3 \pm 4.3$ & $0.95 \pm 0.13$ \\
\hline Diferencia & $4.2 \pm 15.4$ & $0 \pm 4.7$ & $2.3 \pm 5.2$ & $1.2 \pm 5$ & $0.03 \pm 0.13$ \\
\hline Probabilidad & 0.5703 & 1 & 0.3516 & 0.6086 & 0.6431 \\
\hline
\end{tabular}

\begin{tabular}{|l|c|c|c|c|c|}
\hline & Masa Kg & Talla cm & $\begin{array}{c}\text { Porcent. } \\
\text { Grasa }\end{array}$ & IMC & $\begin{array}{c}\text { Ind. Cintura } \\
\text { Cadera }\end{array}$ \\
\hline Sujeto de estudio & 21 & 21 & 21 & 21 & 21 \\
\hline Pre test & $70.2 \pm 11.8$ & $159.0 \pm 7.2$ & $32.8 \pm 7.0$ & $27.6 \pm 4.0$ & $0.94 \pm 0.05$ \\
Post test & $65.5 \pm 11.5$ & $159.0 \pm 7.2$ & $29.5 \pm 6.2$ & $25.8 \pm 4.2$ & $0.92 \pm 0.05$ \\
\hline Diferencia & $4.8 \pm 11.7$ & $0 \pm 7.2$ & $3.2 \pm 6.6$ & $1.8 \pm 4.1$ & $0.02 \pm 0.05$ \\
\hline Probabilidad & 0.1942 & 1 & 0.1213 & 0.1634 & 0.1593 \\
\hline
\end{tabular}


Hombres y Mujeres

\begin{tabular}{|l|c|c|c|c|c|}
\hline & Masa Kg & Talla cm & $\begin{array}{c}\text { Porcent. } \\
\text { Grasa }\end{array}$ & IMC & $\begin{array}{c}\text { Ind. Cintura } \\
\text { Cadera }\end{array}$ \\
\hline Sujeto de estudio & 30 & 30 & 30 & 30 & 30 \\
Pre test & $72.7 \pm 13.6$ & $160.6 \pm 7.0$ & $31.6 \pm 6.7$ & $27.9 \pm 4.4$ & $0.95 \pm 0.09$ \\
Post test & $68.1 \pm 12.9$ & $160.6 \pm 7.0$ & $28.9 \pm 5.7$ & $26.3 \pm 4.3$ & $0.93 \pm 0.08$ \\
\hline Diferencia & $4.8 \pm 13.2$ & $0 \pm 7.0$ & $3.0 \pm 6.2$ & $1.6 \pm 4.3$ & $0.02 \pm 0.08$ \\
\hline Probabilidad & 0.1842 & 1 & 0.0712 & 0.1511 & 0.2704 \\
\hline
\end{tabular}

Fuente propia

La masa en kilogramos, la talla en centímetros, el porcentaje de grasa, el índice de masa corporal y la índice cintura cadera, se midieron en los hombres, no se encontraron diferencias significativas $(P=0.5703, P=1, P=0.3516, P=0.6086$ y $P=0.6431)$. Sin embargo, en la masa en kg, en el índice de masa corporal y el índice cintura cadera se observan que una de dos personas mejoró al someterse al programa de ejercicios. También, se puede afirmar que dos de cada tres personas que se someten a la intervención, en lo que respecta al porcentaje de grasa, lo disminuyen.

La masa en kilogramos, la talla en centímetros, el porcentaje de grasa, el índice de masa corporal y la índice cintura cadera, se midieron en las mujeres, y no se encontraron diferencias significativas $(P=0.1942, P=1, P=0.1213, P=0.1634$ y $P=0.1593$ ). Sin embargo, en la masa en kilogramos, el porcentaje de grasa, el índice de masa corporal y la índice cintura cadera, se demuestra que unas de dos personas mejoraron al someterse a la intervención.

La masa en kilogramos, la talla en centímetros, el porcentaje de grasa, el índice de masa corporal y la índice cintura cadera, se midieron en los hombres y mujeres, no se encontraron diferencias significativas.

La masa en $\mathrm{kg}$ posee una $\mathrm{P}=0.1842$, sugiriendo que 5 de 6 personas que se someten a la intervención disminuyen la masa en $\mathrm{kg}$. La talla en cm se mantuvo, el porcentaje de grasa posee una probabilidad de $P=0.0712$, lo que significa que existe una tendencia fuerte a que 14 de 15 personas que se someten a la intervención disminuyen dicho componente.

Con respecto al índice de masa corporal se encuentra una $\mathrm{P}=0.1511$, lo que significa que existe la probabilidad de que 6 de cada 7 personas que se someten a la intervención mejoran dicho índice; y lo referido al índice cintura cadera, en el cual se encontró una probabilidad de $P=0.2704$, lo que significa que hay una tendencia significativa de que 36 de 37 personas que se sometan a la intervención mejorarían dicho índice. 
La literatura entrega evidencia con respecto a que el entrenamiento o ejercicios con sobrecarga de poca duración de tiempo puede mejorar la calidad de vida de las personas y disminuir sus factores de riesgo cardiovascular, siendo la población en estudio de interés por sus características etarias, étnicas y metabólicas. Los beneficios de mejorar la resistencia muscular y masa magra con el entrenamiento de alta intensidad son viables para los pacientes mayores con diabetes tipo II.

Cuadro 4. Volumen máximo de oxígeno, el índice de inspiración y el índice de Ruffier en hombres, mujeres e integrado; antes y a las 18 semanas de la aplicación del programa de ejercicio físico y educación diabetológica.

\begin{tabular}{|l|c|c|c|}
\hline \multicolumn{3}{|c|}{ Hombres } \\
\hline Sujeto de estudio & 9 & Inspiración & I. Rufier \\
\hline Pre test & $26.8 \pm 4.3$ & 9 & 9 \\
\hline Post test & $41.6 \pm 2.0$ & $2094.4 \pm 727$ & $8.9 \pm 1.9$ \\
\hline Diferencia & $14.9 \pm 3.4$ & $2450 \pm 648$ & $5.2 \pm 1.2$ \\
\hline Probabilidad & 0.0001 & $355.6 \pm 689$ & $3.7 \pm 1.6$ \\
\hline
\end{tabular}

Mujeres

\begin{tabular}{|l|c|c|c|}
\hline & Vo2 Max & Inspiración & I. Rufier \\
\hline Sujeto de estudio & 21 & 21 & 21 \\
\hline Pre test & $29.1 \pm 3.3$ & $1769 \pm 301.9$ & $9.6 \pm 3.7$ \\
\hline Post test & $40.4 \pm 2.2$ & $2147.6 \pm 240$ & $6.0 \pm 1.8$ \\
Diferencia & $11 . .3 \pm 2.8$ & $378.6 \pm 272.7$ & $3.6 \pm 2.9$ \\
\hline Probabilidad & 0.0001 & 0.0001 & 0.0005 \\
\hline
\end{tabular}

Hombres y Mujeres

\begin{tabular}{|l|c|c|c|}
\hline & Vo2 Max & Inspiración & I. Rufier \\
\hline Sujeto de estudio & 9 & 9 & 9 \\
\hline Pre test & $26.8 \pm 3.7$ & $1866.7 \pm 481.3$ & $9.4 \pm 3.2$ \\
\hline Post test & $40.8 \pm 2.2$ & $2238.3 \pm 418.9$ & $5.8 \pm 1.7$ \\
\hline Diferencia & $12.4 \pm 3.1$ & $371.7 \pm 451.1$ & $3.6 \pm 2.6$ \\
\hline Probabilidad & 0.0001 & 0.0023 & 0.0001 \\
\hline
\end{tabular}

Fuente propia

El volumen máximo de oxígeno y el índice de Ruffier se midieron en los hombres, se hallaron diferencias significativas ( $P=0.0001$ y $P=0.0001$ ). No obstante, en el índice de inspiración no se presentó un cambio significativo $(P=0.2896)$, pero demuestra una tendencia fuerte, enunciando que 3 de 4 personas que se someten al estudio mejoran su capacidad vital. 
El volumen máximo de oxígeno, el índice de inspiración y el índice de Ruffier, se midieron en las mujeres, se encontraron diferencias significativas $(P=0.0001$, $\mathrm{P}=0.0001$ y $\mathrm{P}=0.0005$ ). Lo que significa que dichos componentes enunciados mejoraron significativamente.

El volumen máximo de oxígeno, el índice de inspiración y el índice de Ruffier, se midieron en los hombres y mujeres, se encontraron diferencias significativas $(P=0.0001, P=0.0023$ y $P=0.0001)$. Lo que significa que dichos componentes enunciados mejoraron significativamente.

Cuadro 5. Rapidez de pensamiento y autoestima en hombres, mujeres e integrado; antes y a las 18 semanas de la aplicación del programa de ejercicio físico y educación diabetológica.

Hombres

\begin{tabular}{|l|c|c|}
\hline \multicolumn{1}{|c|}{ Rap. Pensam. } & Autoestima \\
\hline Sujeto de estudio & 9 & 9 \\
Pre test & $54.3 \pm 29.5$ & $31.8 \pm 3.6$ \\
Post test & $76.2 \pm 18.3$ & $35.7 \pm 4.4$ \\
Diferencia & $21.9 \pm 24.6$ & $3.9 \pm 4.0$ \\
Probabilidad & 0.0769 & 0.0556 \\
\hline
\end{tabular}

Mujeres

\begin{tabular}{|l|c|c|}
\hline & Rap. Pensam. & Autoestima \\
\hline Sujeto de estudio & 21 & 21 \\
\hline Pre test & $54.7 \pm 21.6$ & $28.5 \pm 7.1$ \\
Post test & $81.1 \pm 11.5$ & $39.5 \pm 5.1$ \\
\hline Diferencia & $26.4 \pm 17.3$ & $11.0 \pm 6.2$ \\
\hline Probabilidad & 0.0001 & 0.0001 \\
\hline
\end{tabular}

Hombres y Mujeres

\begin{tabular}{|l|c|c|}
\hline \multicolumn{1}{|c|}{ Rap. Pensam. } & Autoestima \\
\hline Sujeto de estudio & 30 & 30 \\
Pre test & $54.6 \pm 23.7$ & $29.5 \pm 6.4$ \\
Post test & $76.6 \pm 13.7$ & $38.3 \pm 5.1$ \\
Diferencia & $25.1 \pm 19.4$ & $8.9 \pm 5.8$ \\
Probabilidad & 0.0001 & 0.0001 \\
\hline
\end{tabular}

Fuente propia 
La rapidez de pensamiento y autoestima se midieron en los hombres, se encontraron diferencias significativas ( $P=0.0769$ y $P=0.0556)$. Lo que significa que dichos componentes enunciados mejoraron significativamente.

La rapidez de pensamiento y la autoestima se midieron en las mujeres, se encontraron diferencias significativas $(P=0.0001$ y $P=0.0001)$. Lo que significa que dichos componentes enunciados mejoraron significativamente.

La rapidez de pensamiento y la autoestima se midieron en los hombres y mujeres, se encontraron diferencias significativas: $\mathrm{P}=0.0001$ y $\mathrm{P}=0.0001$.

\section{DISCUSIÓN}

Al igual que en otras investigaciones, fueron evidentes los beneficios adicionales del ejercicio en el paciente con Diabetes mellitus tipo II.

1. Existen suficientes pruebas en la literatura que justifican la indicación del ejercicio físico, como una herramienta terapéutica efectiva en la prevención y el tratamiento de la DM2 (Sánchez, T. ,2011 y Moreno-Bayona, J. ,2016).

2. La relación positiva entre la circunferencia abdominal, la obesidad central, la resistencia a la insulina, el síndrome metabólico y las enfermedades cardiovasculares convierte la reducción de esa variable en uno de los objetivos importantes en el tratamiento de la DM2. Los pacientes que integraron el grupo denominado experimental mostraron una reducción no significativa de la CC y del IMC de forma indirecta al lograr perder peso durante la realización del programa de ejercicios físicos, hecho este, que se lograría de forma significativa con una exposición más prolongada al programa de ejercicios. Sin embargo, con las cifras tensionales no ocurrió lo mismo en este grupo, cabría destacar que estas personas no mostraron cifras elevadas en la valoración inicial de la investigación, pero si se logró una disminución significativa de las mismas al finalizar el programa aplicado y comparar los valores con el grupo control.(Quílez, P. ,2015) y (Arias-Vázquez, P. ,2015): 


\begin{tabular}{|l|l|}
\hline \multicolumn{1}{|c|}{ Parámetro } & \multicolumn{1}{c|}{ Efecto } \\
\hline Cardiovascular & Disminuye \\
\hline Riesgo Cardiovascular & Disminuye/ se mantiene \\
\hline Capacidad aeróbica o nivel de fitness & Disminuye \\
\hline Frecuencia cardiaca en reposo & Disminuye \\
\hline Presión arterial & Disminuye \\
\hline Mortalidad cardiovascular y por todas las causas & \\
\hline Lípidos y lipoproteínas & Aumenta \\
\hline HDL & Disminuye \\
\hline LDL & Disminuye \\
\hline VLDL & Disminuye \\
\hline Colesterol total & \\
\hline Antropometría & Disminuye \\
\hline Peso & Disminuye \\
\hline Masa grasa & \\
\hline Metabólico & Aumenta \\
\hline Sensibilidad a la insulina & \\
\hline Psicosocial & Aumenta \\
\hline Autoconcepto y autocuidado & Disminuye \\
\hline Depresión & Disminuye \\
\hline Ansiedad & Disminuye \\
\hline Respuesta al stress psicológico & Aumenta \\
\hline Calidad de vida & \\
\hline
\end{tabular}

En el presente estudio de investigación los resultados fueron similares a otros estudios que valoraron el ejercicio físico para el manejo de pacientes con Diabetes mellitus, reportando lo siguiente:

1. Disminución de los valores de la Glucosa, hemoglobina glicosilada, ácido úrico y aumento de la hemoglobina en el post test.

2. Se evidenció disminución del riesgo coronario al reportar descenso del Colesterol LDL, Triglicéridos y colesterol total, también se observó en el presente estudio disminución de la masa corporal e índice de cintura cadera.

3. En relación al VO2 Max presentó un aumento significativo mejorando la calidad de vida del paciente, disminuyendo el Índice de Rufier.

4. La rapidez de pensamiento y la autoestima mejoró en los pacientes diabéticos posterior al programa de ejercicio físico $(20,21)$. 


\section{BIBLIOGRAFÍA}

Augusto. L. (2012). Historia de la diabetes en la antigüedad hasta la insulina. Recuperado de http://www.sinazucar.info/

Chudyk A, Petrella (2011) RJ. Effects of exercise on cardiovascular risk factors in type

2 diabetes: a meta-analysis. Diabetes Care 34(5):1228-37

Kwon HR, Min KW, Ahn HJ, Seok HG, Koo BK, Kim HC (2010). Effects of aerobic exercise on abdominal fat, thigh muscle mass and muscle strength in type 2 diabetic subject. Korean Diabetes J. 34(1):23-31, 2010

Márquez, JJ Arabia. Suarez G, Ramón y Márquez Tróchez, J. (2012). (12 de febrero de 2012). El ejercicio en el tratamiento de la diabetes Mellitus Tipo 2. Volumen 48. Recuperado de: http://www.scielo.org.ar/pdf/raem/v49n4/v49n4a06.pdf Aguilar Rodríguez, Yissel. Sánchez, Vicente. Llaguno Pérez, Guillerme y Col. (2012). Efecto del ejercicio físico y el control metabólico y factores de riesgo de pacientes con diabetes mellitus tipo 2: estudio cuasi experimental. Recuperado de: www.medwave.cl/link.cgi/Medwave/Estudios/Investigacion/5547

American Diabetes Association. (2015). Diagnosis and classification of diabetes mellitus. Disponible en http://www.sciencedirect.com/science/article/pii/S0300893202 766463

Calles, Diego. (2011). Efectividad de un programa de ejercicios físicos terapéuticos aplicado a pacientes con diabetes mellitus tipo II, que asisten al Instituto Ecuatoriano de Seguridad Social de Guayas, Ecuador. Tesis de maestría. Universidad de Ciencia de la Cultura Física Manuel Fajardo. La Habana.

Ibáñez, Ricardo. (2009). Orientaciones metodológicas para la preparación del Profesor de cultura física en la atención a embarazadas con diabetes mellitus (Tesis de maestría). Universidad de Ciencias de la Cultura Física Manuel Fajardo. Santa Clara.

Morales, Alejandro. (2013). El ejercicio físico en el diabético. Universidad de Ciencias de la Cultura Física. Editorial Pueblo y Educación: La Habana.

Sanchez, Tarym. (2011). Plan de ejercicios físicos profilácticos y terapéuticos para la rehabilitación de las personas adultas mayores con diabetes mellitus tipo II en el contexto domiciliario (Tesis de maestría). Universidad de Ciencias de la Cultura Física. La Habana.

Moreno-Bayona, Jesus y Cote Mogollón, Fernando (2016): Ejercicio intermitente de alta intensidad como estrategia terapéutica para diabéticos tipo 2 residentes en alturas intermedias. 42 (4). Recuperado de: http://www.revsaludpublica.sld.cu/index.php/spu/article/viewFile/869/803. 
Márquez Arabia JJ, Ramón Suárez G, Márquez Tróchez J (2012): El ejercicio en el tratamiento de la diabetes mellitus tipo 2. Revista Argentina de Endocrinología y Metabolismo 48 (4), (203-212) Recuperado de: http://www.scielo.org.ar/pdf/raem/v49n4/v49n4a06.pdf.

Organización Mundial de la Salud (2016): Diabetes. OMS. Recuperado de: http://www.who.int/mediacentre/factsheets/fs312/es/

Quílez Llopis, Pablo (2015): Control glucémico a través del ejercicio físico en pacientes con diabetes mellitus tipo 2. Nutrición Hospitalaria 31 (4), (1465-1472).

Arias-Vázquez. Pedro (2015): Actividad física en Diabetes Mellitus tipo II, un elemento terapéutico eficaz: revisión del impacto clínico. Actividad física en Diabetes Mellitus tipo II. DUAZARY. 12 (2), (147-156). Recuperado de: //dialnet.unirioja.es/descarga/articulo/5156567.pdf. 